\title{
Grupos de Creación y Gestión del Conocimiento en Red en un Programa de Perfeccionamiento en Docencia Universitaria
}

\section{Online Knowledge Creation and Management Groups in a University Teaching Training Program}

\author{
Anna Díaz-Vicario \\ Universitat Autònoma de Barcelona. Barcelona, España \\ anna.diaz@uab.cat \\ Joaquín Gairín Sallán \\ Universitat Autònoma de Barcelona. Barcelona, España \\ joaquin.gairin@uab.cat
}

\begin{abstract}
Resumen
Los grupos de creación y gestión del conocimiento son un importante recurso para el desarrollo profesional, dando respuesta a las necesidades de actualización y mejora constante de la realidad profesional. En el marco de un Programa de Perfeccionamiento en Docencia Universitaria dirigido a profesorado de la Universidad de Tarapacá (Chile), se han desarrollado seis grupos de Creación y Gestión del Conocimiento (CGC) en Red con la participación de cerca de 250 profesores. Los grupos, como actividad transversal al programa formativo, facilitan el intercambio de conocimiento, la reflexión sobre la práctica y la búsqueda colectiva de soluciones a problemas compartidos sobre docencia y gestión universitaria. El desarrollo de la experiencia constata una manera de vincular grupos de CGC utilizando la web social en acciones de formación permanente; también, que su utilización no sólo contribuye al desarrollo profesional y personal del profesorado, sino que también favorece el desarrollo institucional. Difundir los productos generados en el seno de los grupos de CGC entre los responsables institucionales puede servir a su diseminación y consolidación, así como de punto de partida para el establecimiento de líneas estratégicas en la universidad.
\end{abstract}

Palabras clave

Creación y Gestión del Conocimiento, Formación continua, Profesorado universitario, Entornos Virtuales de Aprendizaje, Web social

\begin{abstract}
Knowledge Creation and Management Groups are an important resource for professional development, addressing the needs for continuing professional development and regularly improvement of the professional context. In the context of a University Teaching Training Program, addressed to academic and teaching staff of the Universidad of Tarapacá (Chile), has been developed six online Knowledge Creation and Management Groups. Nearly 250 academic and teaching staff participated in the experience. The groups, as a cross-curricular activity, allow knowledge sharing, reflection on practices, and the search for solutions about shared problems related to teaching practices and higher education management. The experience shows that is possible bind online Knowledge Creation and Management Groups and continuous training activities together, and that their use not only contributes to the professional and personal development of academic and teaching staff but also assist the institutional development. Share the products created inside the Knowledge Creation and Management Groups with the academic authorities can assure the consolidation of the practices and support the establishment of strategic guidelines.
\end{abstract}

Key words

Creation and Management of Knowledge, Life-long learning, Academic and teaching staff, Virtual learning environments, Social web 


\section{Introducción}

Las Tecnologías de la Información y la Comunicación (TIC) o sus formatos posteriores (TAC: Tecnologías del Aprendizaje y el Conocimiento; TEP: Tecnologías para el Empoderamiento y la Participación) forman ya parte del normal desarrollo de cualquier programa formativo. Casi la totalidad de programas hacen referencia al aula Moodle, al campus virtual o al correo electrónico. Habitualmente se emplean como espacios de comunicación entre estudiantes y profesores, para el intercambio de archivos y el anuncio de actividades, aunque, actualmente, se ha avanzado hacia nuevos usos que permiten pasar del mero intercambio de información hacia la creación colaborativa de conocimiento.

Las herramientas que pueden emplearse para facilitar la comunicación, el intercambio, la reflexión y la construcción colaborativa de conocimiento son múltiples y variadas: foros, chats, wikis, blogs, documentos compartidos, etc. Y es que las llamadas groupware o tecnologías para el trabajo en grupo facilitan que los usuarios puedan comunicarse, compartir y coordinar la información de manera más ágil y rápida. Las tecnologías puestas al servicio de la formación facilitan la discusión, la búsqueda de significados compartidos y la construcción colectiva de conocimiento. Permiten, asimismo, lograr aprendizajes más significativos, al forzar al estudiante a pensar y comunicar sus ideas.

Aunque la presencia de las TIC en la enseñanza y las experiencias de formación virtual ya se han asentado a nivel de los estudios de Grado y Postgrado (aunque no en todos los contextos universitarios), no sucede lo mismo en el caso de las acciones de formación permanente del profesorado universitario. La oferta formativa sigue siendo mayoritariamente presencial, en formato de cursos y talleres, siendo menores las experiencias basadas en enfoques bimodales o de blended learning. Asimismo, están muy centradas en la planificación, en la evaluación y el uso de las TIC, siendo pocos los programas que enfaticen en la reflexión sobre docencia y gestión universitaria (Espejo \& González, 2014).

En el contexto iberoamericano, una gran parte de los docentes cuentan con escasa preparación didáctica. El último informe del estado de la Educación Superior en Iberoamérica (Brunner \& Miranda, 2016) señala que el profesorado universitario, entre ellos el chileno, cuenta con limitadas oportunidades de capacitación y perfeccionamiento para abordar el reto que supone el formar a las nuevas generaciones.

Sistemas universitarios más aventajados, como el español, y con una larga tradición en el desarrollo y perfeccionamiento de su cuerpo académico, subrayan la necesidad de que la formación continua se imbrique en las necesidades específicas de las organizaciones y de los profesionales con el objetivo de mejorar tanto la calidad de la enseñanza como el funcionamiento de las instituciones. Informes, como el del Ministerio de Educación, Cultura y Deporte de España (MEC, 2015) que recoge recomendaciones formuladas por organismos e instituciones nacionales e internacionales, indican que más allá de mejorar las competencias docentes, es una prioridad ofrecer al profesorado entornos compartidos de reflexión y aprendizaje, estimulando la puesta en común de estrategias que den respuesta a necesidades concretas y que permitan mejorar la práctica docente.

Grupos de creación y gestión del conocimiento en red en un programa de perfeccionamiento en docencia universitaria. Anna Díaz-Vicario y Joaquín Gairín Sallán 
En la misma línea, estudios como el de Duţă y Rafailă (2014) sostienen que la formación del profesorado universitario debe estimular la reflexión individual y colectiva para resolver situaciones problemáticas de la práctica docente. Bozu e Imbernón (2016) señalan como estrategias para favorecer la reflexión del profesorado universitario sobre la propia práctica: la creación de redes formales e informales de intercambio de experiencias y de difusión de iniciativas, la promoción de grupos de innovación, el aprovechamiento de los espacios naturales de aprendizaje, etc. Y es que se plantea que acciones formativas individuales y pasivas son inadecuadas para preparar al profesorado en la integración de habilidades que los estudiantes necesitan para progresar con éxito tanto en su formación como en su futuro lugar de trabajo (Stewart, 2014).

Se justifica así apostar por una formación permanente del profesorado universitario que, más allá de la formación en competencias docentes, vele por el establecimiento de una cultura de aprendizaje colaborativo que conduzca hacia la creación de comunidades de profesionales con diferente nivel de pericia y conocimiento (Puntman \& Borko, 2000). Es el momento de que los servicios de formación apuesten por generar entornos colaborativos de aprendizaje que permitan al profesorado experimentar, reflexionar y aprender de otros mediante la interacción, más cuando ya hace años que diversos autores señalan que aprendemos más colaborando, participando e interactuando con otros (Avello \& Duart, 2016; Stewart, 2014; Trust, Krutka, \& Carpenter, 2016; Wenger, 1998). Las TIC pueden apoyar todo este proceso, tal y como revelan diversos estudios (Charband \& Jafari, 2016; Gairín \& Rodríguez-Gómez, 2012; López, Peón \& Ordás, 2009).

El presente trabajo describe la experiencia de creación de grupos de Creación y Gestión del Conocimiento (CGC, en adelante) en Red dentro de un Programa de Perfeccionamiento en Docencia Universitaria semipresencial de la Universidad de Tarapacá (Chile), en colaboración con la Universidad Autónoma de Barcelona (España). La formación semipresencial se orienta a favorecer un ambiente formativo centrado en el estudiante y conectado con la práctica profesional. Entre las actividades a distancia vinculados a los 33 cursos de formación de 50 horas realizados, se han desarrollado 6 grupos de CGC en Red de entre siete y ocho semanas de duración, con la participación inicial de 424 docentes universitarios con una carga total de trabajo de entre 3 y 5 horas para cada uno de ellos.

El interés de la experiencia radica en el hecho de que los grupos de CGC promueven la relación y convivencia de profesores que, aun y trabajar en la misma universidad, proceden de Facultades, Departamentos y Áreas de especialización distintas que, en algunos casos, se encuentran físicamente ubicados en diferentes sedes de la Universidad. Integrar los grupos de CGC en Red como parte del programa formativo ha permitido dotar al profesorado de un espacio de trabajo colaborativo intrainstitucional e interprofesional en el que pensar, compartir y buscar respuestas y soluciones a temas de interés común, rompiendo las barreras del espacio, el tiempo y la especialidad profesional.

Los siguientes apartados clarifican conceptos clave y presentan los antecedentes teóricos en los que se fundamenta la propuesta. Seguidamente, se describe el contexto y

Grupos de creación y gestión del conocimiento en red en un programa de perfeccionamiento en docencia universitaria. Anna Díaz-Vicario y Joaquín Gairín Sallán 
antecedentes que dieron lugar a la propuesta de conformar grupos de CGC en Red y se explica la experiencia llevada a cabo, analizando tanto las condiciones clave que han garantizado el éxito de la experiencia como las oportunidades de mejora.

\section{Los Grupos de Creación y Gestión de Conocimiento (CGC)}

Actualmente, desde diferentes contextos teóricos y prácticos se plantea la necesidad de configurar una cultura profesional basada en la colaboración, es decir, una cultura que tenga en cuenta, como elementos centrales: la comunicación, el trabajo compartido, el intercambio de prácticas profesionales, la reflexión colectiva y la búsqueda de soluciones a problemáticas comunes. Particularmente, la cultura colaborativa debe darse en las instituciones dedicadas a la formación y que se caracterizan por un alto individualismo y por el modo celular con el que orientan su trabajo (Ali, Ghoneim, \& Al Roubaie, 2014). Tiene sentido plantear en ellas el desarrollo de grupos de creación y gestión del conocimiento que integren distintos miembros, impulsen estrategias de trabajo compartidas, se identifiquen con el proyecto colectivo y creen una cultura de trabajo común (Gairín \& Díaz, 2011).

La CGC se define como "un proceso sistemático que permite crear, recopilar, organizar, difundir, usar y explotar el conocimiento para mejorar el desempeño de las personas en el contexto de cualquier tipo de organización" (García \& Gómez, 2015, p. 15). En este sentido, las prácticas de CGC en instituciones de educación superior suponen considerar todas aquellas actividades encaminadas a la adquisición, captura, almacenamiento, diseminación y utilización del conocimiento por parte del profesorado y de los gestores institucionales, permitiendo poner en valor el conocimiento de los propios profesionales, mejorar los programas educativos y, en consecuencia, mejorar el rendimiento y resultados de la institución (Ali, Ghoneim, \& Al Roubaie, 2014; Cantón \& Ferrero, 2014; Fidalgo-Blanco, Sein-Ecjaluce, \& García-Peñalvo, 2014).

Se trata de generar espacios para la colaboración e interacción de los profesionales, para que individuos con los mismos intereses, backgrounds y objetivos puedan compartir su conocimiento formulando preguntas, proponiendo respuestas y debatiendo cuestiones de interés compartido (Charband \& Jafari, 2016), haciendo posible que el aprendizaje individual del profesorado se trasforme en aprendizaje colectivo que pueda ser puesto al alcance de toda la institución (Macià \& García, 2016). Las TIC pueden facilitar el proceso, utilizando foros de discusión, listas de distribución, blogs, wikis u otras herramientas, teniendo en cuenta que cada una de ellas puede servir a un propósito distinto (ver Tabla 1):

Tabla 1. Técnicas y tecnologías para la CGC (Rodríguez-Gómez, 2009, citado por Gairín \& Rodríguez-Gómez 2012, p. 280).

\begin{tabular}{|c|c|c|}
\hline Procesos de CGC & Objetivo & Técnicas y tecnologías \\
\hline $\begin{array}{l}\text { Localización, } \\
\text { almacenaje y acceso }\end{array}$ & $\begin{array}{l}\text { Almacenar conocimiento } \\
\text { organizativo, } \\
\text { incrementando así la } \\
\text { memoria organizativa y } \\
\text { facilitando su localización, } \\
\text { acceso y posterior difusión }\end{array}$ & $\begin{array}{l}\text { Repositorios de conocimiento, } \\
\text { bases de datos, mapas de } \\
\text { conocimiento, páginas amarillas, } \\
\text { directorios de conocimiento, } \\
\text { motores de búsqueda, agentes } \\
\text { inteligentes, auditorías de } \\
\text { conocimiento, buenas prácticas, }\end{array}$ \\
\hline
\end{tabular}

Grupos de creación y gestión del conocimiento en red en un programa de perfeccionamiento en docencia universitaria. Anna Díaz-Vicario y Joaquín Gairín Sallán

Página 4 de 20 


\begin{tabular}{|c|c|c|}
\hline & & $\begin{array}{l}\text { educción de conocimiento, } \\
\text { ontologías, reclutamiento, gestor } \\
\text { de contenidos, social } \\
\text { bookmarking }\end{array}$ \\
\hline Creación & $\begin{array}{l}\text { Descubrir y crear nuevos } \\
\text { patrones/conocimientos }\end{array}$ & $\begin{array}{l}\text { Minería de datos, CoP, } \\
\text { fórums/debates, herramientas } \\
\text { estadísticas, representación } \\
\text { gráfica, tecnologías de } \\
\text { simulación, formación, } \\
\text { encuentros de asistencia y ayuda, } \\
\text { lluvia de ideas, mentoría, } \\
\text { reuniones de revisión, social } \\
\text { bookmarking }\end{array}$ \\
\hline Difusión/compartir & $\begin{array}{l}\text { Difusión del conocimiento } \\
\text { organizativo disponible, } \\
\text { haciendo posible que las } \\
\text { personas adecuadas } \\
\text { dispongan del } \\
\text { conocimiento adecuado }\end{array}$ & $\begin{array}{l}\text { Correo electrónico, CoP, } \\
\text { boletines electrónicos, listas de } \\
\text { distribución, foros de debate, } \\
\text { chat, mensajería instantánea, } \\
\text { wikis, blogs, podcast, } \\
\text { videoconferencias, groupware, } \\
\text { intranets, extranets, portales de } \\
\text { conocimiento, análisis de } \\
\text { contenido, análisis de redes } \\
\text { sociales, buenas prácticas, } \\
\text { formación, historias de } \\
\text { conocimiento/narrativas, } \\
\text { encuentros de asistencia y ayuda, } \\
\text { interacción cara a cara, world- } \\
\text { café, RRS }\end{array}$ \\
\hline Utilización & $\begin{array}{l}\text { Facilitar la integración y } \\
\text { aplicación del } \\
\text { conocimiento organizativo }\end{array}$ & $\begin{array}{l}\text { Sistemas de expertos, árboles de } \\
\text { decisión, workflow systems, } \\
\text { mapas de conocimiento }\end{array}$ \\
\hline
\end{tabular}

Habitualmente, el profesorado no es consciente de lo qué sabe, no dispone de espacios adecuados para compartir o del tiempo necesario. Murua (2014) nos recuerda que,

Si bien compartir es tan antiguo como el hombre [...] cabe preguntarse hasta qué punto el profesorado participa en este tipo de redes, si desarrolla el interés por aprender de los pares, si formaliza, documenta y comunica su conocimiento tácito. (p. 2)

Los gestores institucionales deben generar dinámicas grupales que promuevan que el profesorado crea, comparta, difunda e interiorice el conocimiento, puesto que la colaboración entre profesionales (Gairín \& Díaz, 2011, p. 16):

- Ayuda a superar el individualismo al que lleva muchas veces la actividad profesional.

- Aumenta la potencia creativa del equipo resultante.

- Encauza la actividad reflexiva sobre la propia actividad profesional, con connotaciones de mejora personal, colectiva y profesional. 
- Posibilita el compartir el patrimonio de habilidad y experiencia que existe en la organización.

- Fortalece la actividad colectiva y la consideración de las organizaciones como comunidades formativas y de desarrollo profesional.

La creación de grupos de CGC en Red, como una agrupación de profesionales que se organiza para involucrarse en un proyecto común y que trabajan cooperativamente y en base a principios de participación abierta y flexible, puede hacerlo posible. Los grupos en Red, frente a los grupos presenciales:

- Facilitan la comunicación.

- Posibilitan crear espacios para compartir y debatir ideas y creencias personales que de otro modo no se producirían.

- Hacen posible que la discusión de cualquier idea se dé entre un mayor número de interlocutores.

- Aumenta la velocidad en la transmisión de la información.

La web social tiene el potencial de responder a las necesidades individuales y colectivas del profesorado (Macià \& García, 2016), pero debe evitarse caer en el error de generar espacios virtuales que acaben convirtiéndose en simples almacenes de información (Martínez \& Cambiano, 2003). Tener acceso a un entorno de trabajo colaborativo en red con un alto grado de usabilidad, navegación, estructuración, contenido y herramientas sociales, y que dé respuesta a las necesidades del grupo, es crucial para motivar la participación del profesorado (Macià \& García, 2016). Por tanto, cualquier organización educativa que se plantee poner en marcha grupos de CGC virtuales debe poner al alcance de los profesionales los medios necesarios y las condiciones favorables que lo hacen preciso (Gairín, 2011).

Los procesos comunicativos, la interdependencia positiva entre los miembros y la motivación son considerados aspectos cruciales que pueden marcar el éxito o el fracaso de los grupos de CGC en Red, y que reiteradamente son identificados en la literatura (Charband \& Jafari, 2016; Macià \& García, 2016; Gairín, 2011). En este sentido, Knight (2011, citado por Stewart, 2014, p. 29) enumera siete principios que es necesario tomar en cuenta cuando queremos generar aprendizaje profesional (ver Tabla 2):

Tabla 2. Descripción de los principios de colaboración para el aprendizaje grupal.

\begin{tabular}{|l|l|}
\hline \multicolumn{1}{|c|}{ Principio } & \multicolumn{1}{c|}{ Descripción } \\
\hline Igualdad & $\begin{array}{l}\text { Los profesores reciben inputs que van más allá de los aspectos } \\
\text { planificados para las actividades de desarrollo profesional. }\end{array}$ \\
\hline Elección & Los profesores eligen qué y cómo aprenden. \\
\hline Voz & El aprendizaje faculta y respeta la voz de los profesores. \\
\hline Reflexión & $\begin{array}{l}\text { La reflexión es reconocida como parte integral del aprendizaje. Se } \\
\text { garantiza el diálogo auténtico. }\end{array}$ \\
\hline Práctica & El aprendizaje se vincula con prácticas cotidianas. \\
\hline Reciprocidad & La participación es recíproca: todos aportan y todos reciben. \\
\hline
\end{tabular}

Las experiencias de CGC en instituciones de educación superior son limitadas, tal como evidencia el estudio de Ali, Ghoneim, y Al Roubaie (2014) por la falta de sistemas y

Grupos de creación y gestión del conocimiento en red en un programa de perfeccionamiento en docencia universitaria. Anna Díaz-Vicario y Joaquín Gairín Sallán 
políticas para proteger los activos intelectuales del profesorado, la naturaleza individualista de los académicos, la complejidad de la estructura organizativa de las instituciones de educación superior, entre otros. Los autores abogan por extender prácticas de CGC basadas en la cultura colaborativa, como la que aquí se presenta.

\section{Contexto de desarrollo de la experiencia}

El profesorado universitario necesita formación específica para desempeñar de la mejor manera posible la función docente (Bozu \& Imbernón, 2016). Las instituciones de educación superior cuentan con estructuras organizativas específicas orientadas a este propósito, dando también respuestas a los intereses y necesidades del modelo académico que deben implementar.

El Centro de Innovación y Desarrollo de la Docencia (CIDD) de la Universidad de Tarapacá (UTA, Chile) inició, al respecto, un plan de perfeccionamiento docente que tiene por objetivo mejorar las prácticas docentes y ayudar en la implementación de los modelos educativos y pedagógicos aprobados en los años 2011 y 2017, respectivamente. Como parte de la estrategia, la Universidad de Tarapacá firmó en el año 2015 un convenio con el Departamento de Pedagogía Aplicada de la Universitat Autònoma de Barcelona (UAB, España) para realizar el Programa de Perfeccionamiento en Docencia Universitaria. El programa, de dos años y medio de duración y dirigido a un grupo aproximado de 250 profesores universitarios de diferentes áreas y departamentos, persigue un triple objetivo: a) mejorar la docencia; b) incidir en la mejora de los resultados académicos de los estudiantes; y c) cumplir con la exigencia de perfeccionamiento del profesorado, considerando que cada uno de ellos hace varios módulos de formación de los previstos.

El Programa se estructura en 6 líneas de actualización (Socialización del Modelo Educativo Institucional, Métodos de enseñanza-aprendizaje, Modalidades organizativas, Gestión del aprendizaje, Tecnología para la formación, e Investigación en docencia universitaria), que se traducen en 15 Módulos formativos (Aprendizaje Basado en Problemas, El estudio de casos, Diseño curricular de la asignatura, Uso de la tecnología en el aula, etc.). Cada uno de los módulos se concreta en cursos de formación cuya duración inicial sería de una semana, con 36 horas presenciales de clase, 6 horas para planificar la intervención y 8 horas para llevarla a cabo y elaborar el trabajo final.

La formación se orienta a proporcionar herramientas para favorecer un ambiente formativo centrado en el estudiante, conectado a la práctica profesional y orientada a la mejora de ésta, combinando tanto el desarrollo de actividades presenciales como a distancia a través del entorno Moodle.

Entre las actividades de carácter virtual, se contempla la realización de grupos de CGC en Red. Éstos se desarrollan transversalmente a las actividades académicas propias del programa formativo, abriendo diversos grupos en diferentes momentos para hacer posible que todos los profesores inscritos en el programa participen, como mínimo, en uno de los grupos.

Cabe considerar que la sede central de la UTA se encuentra ubicada en Arica, capital de la XV Región, pero que dicha universidad cuenta con una dispersión de instalaciones y

Grupos de creación y gestión del conocimiento en red en un programa de perfeccionamiento en docencia universitaria. Anna Díaz-Vicario y Joaquín Gairín Sallán

Página $\mathbf{7}$ de $\mathbf{2 0}$ 
con otra sede en Iquique, en la Región de Tarapacá. A ambas sedes las separan 310 km de distancia, hecho que dificulta que los académicos puedan reunirse para compartir inquietudes, intercambiar opiniones y llevar a cabo debates colectivos sobre temas vinculados con la docencia y la gestión de la universidad.

Los grupos de CGC, más allá de ser una actividad de carácter académico, suponen una oportunidad única para que el profesorado pueda reflexionar sobre problemas de la práctica diaria que les son comunes. Además, al desarrollarse virtualmente, se rompen las barreras del espacio y el tiempo, haciendo posible que la reflexión individual nutra la reflexión colectiva; es decir, que el profesorado comparta su conocimiento tácito, cree referentes comunes, defina estrategias de análisis de la realidad y proponga acciones específicas que permitan modificarla y mejorarla.

\section{Diseño y desarrollo de la experiencia}

El desarrollo de la experiencia de formación en red basada en grupos de CGC que aquí presentamos parte de los resultados positivos ya observados en experiencias anteriores, pero no vinculadas a programas formativos y desarrolladas en un ámbito territorial más reducido y con un menor número de participantes (Gairín \& Armengol, 2006; Gairín, 2008; Gairín \& Rodríguez-Gómez, 2010). Se basa en tres premisas básicas (Gairín \& Rodríguez-Gómez, 2008):

a. La consideración de los grupos de CGC como una estrategia de formación continua que se vincula a los procesos de mejora interna de las organizaciones.

b. La creencia de que el diálogo, la acción y la solidaridad intergeneracional e intersujetos pueden contribuir a generar un aprendizaje significativo para el que aprende.

c. La capacidad de generar nuevo conocimiento aumenta al compartir aquellos surgidos de la reflexión y estructuración de la propia práctica.

Los grupos de CGC en Red de la UTA se crean en el marco del Programa de Perfeccionamiento en Docencia Universitaria con el objetivo de constituir un espacio de encuentro virtual en el que generar e intercambiar conocimientos y experiencias mediante un proceso colaborativo. La experiencia cuenta con el aval de los responsables institucionales, quienes ven en la misma una oportunidad de conformar comunidades formativas para la reflexión y el diálogo entre académicos.

\subsection{El modelo de referencia}

Los grupos de CGC en Red se basan en el principio de la colaboración entre profesionales. La colaboración puede expresarse y concretar en diferentes formatos según la modalidad, el nivel de implicación y amplitud. En nuestro caso, el formato más adecuado de colaboración para los propósitos pretendidos queda representado en la Figura 1. 


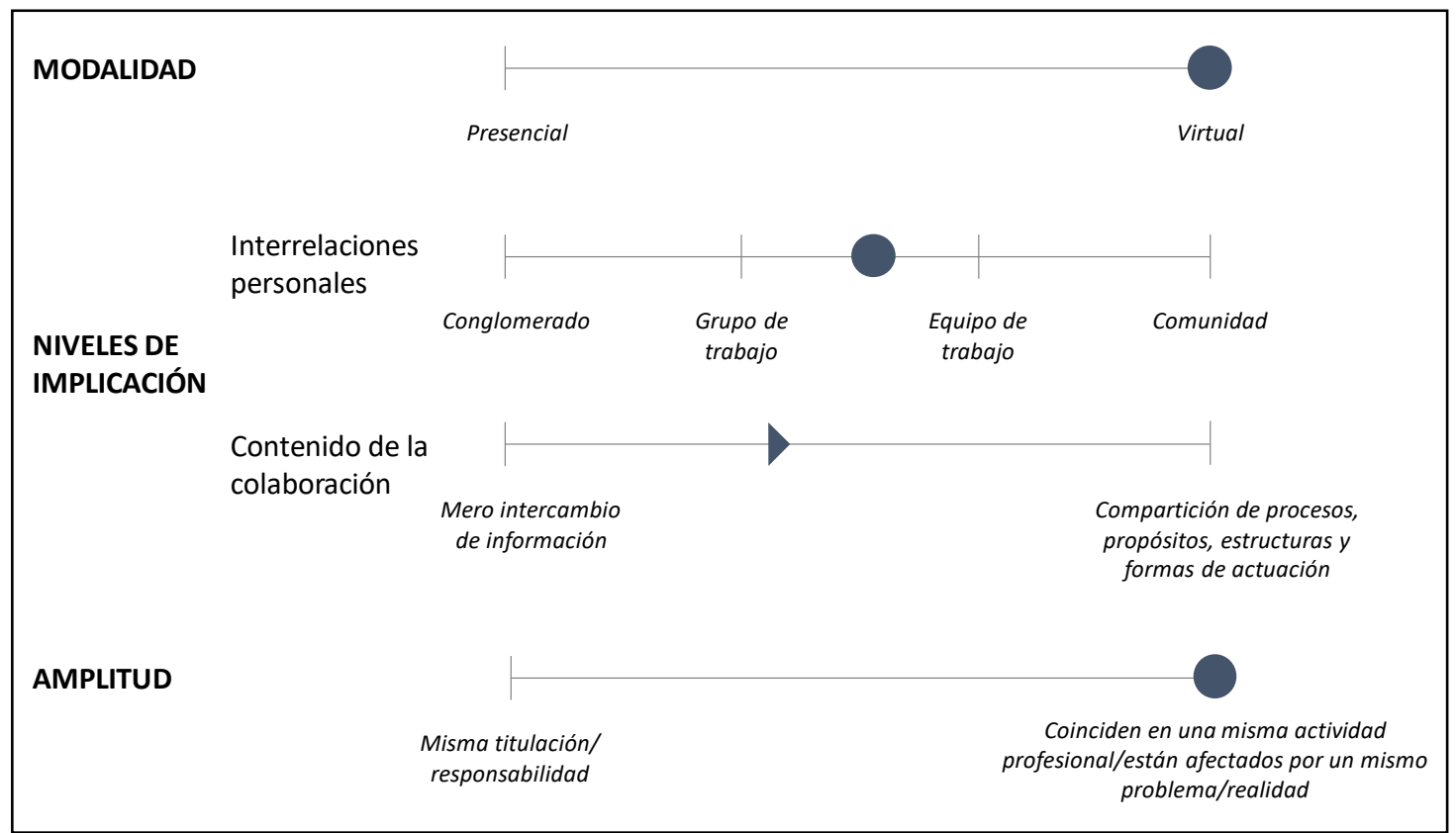

Figura 1. Formato de la colaboración de los grupos de CGC en Red de Tarapacá.

Se establece una modalidad de colaboración virtual en la que profesorado de diferentes áreas, departamentos y facultades coinciden en una misma actividad de carácter formativo-profesional. La comunicación es asincrónica, en lo que a la construcción de los mensajes se refiere, y se pretende que, en el interior de la red, más que un mero intercambio de información, se compartan propósitos y formas de actuación.

El modelo de gestión de conocimiento de los grupos de la UTA se basa en las características del Modelo Accelera de creación y gestión del conocimiento (Gairín, 2008) y que ha sido experimentado con éxito en el contexto español desde el año 2005. Con todo, el modelo ha sido parcialmente adaptado a las características y necesidades del Programa Formativo de Tarapacá (ver Figura 2).

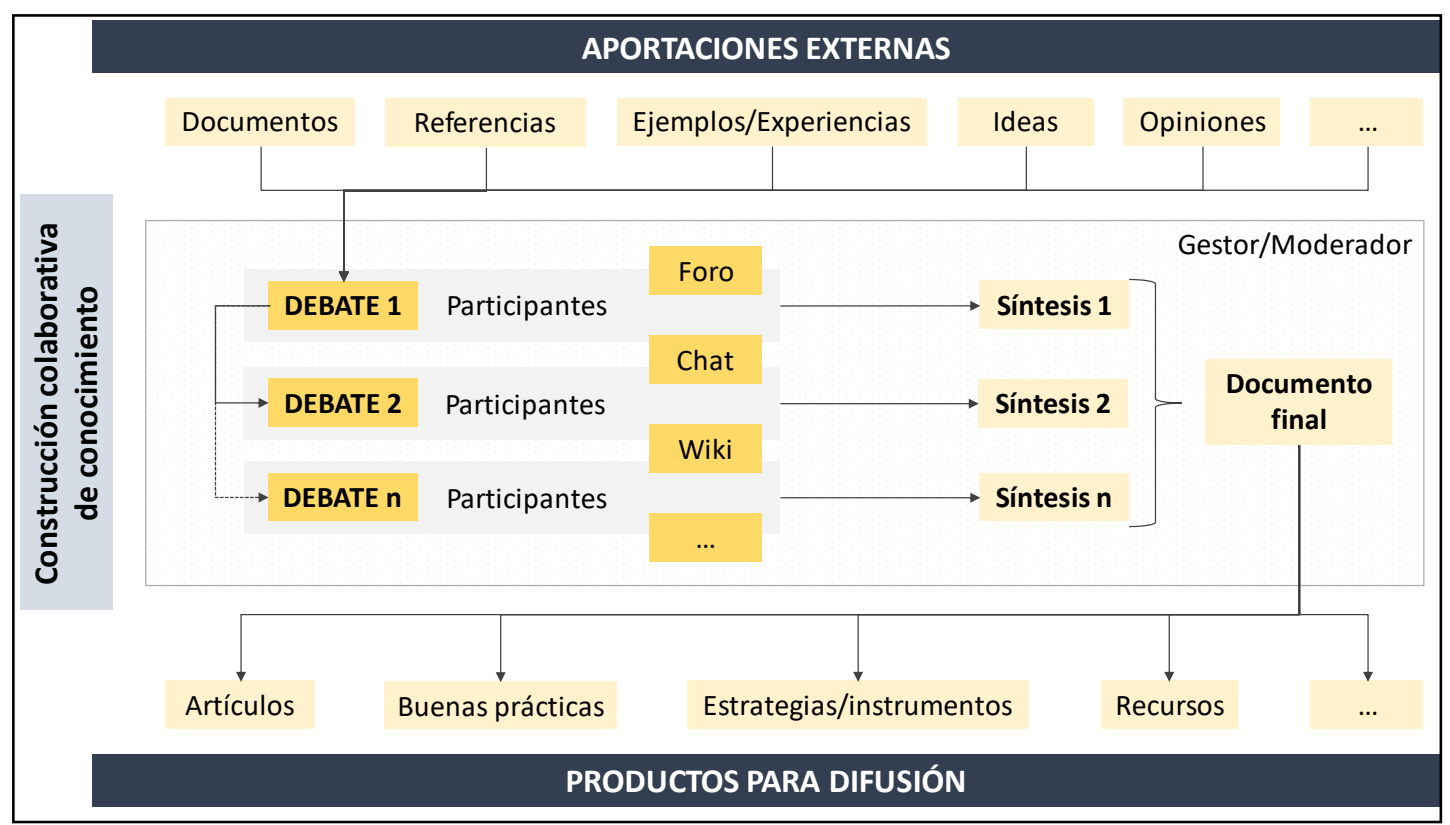

Figura 2. Modelo de gestión del conocimiento (a partir de Gairín, 2008, p. 6).

Grupos de creación y gestión del conocimiento en red en un programa de perfeccionamiento en docencia universitaria. Anna Díaz-Vicario y Joaquín Gairín Sallán 
El núcleo del modelo lo constituye el debate, como estrategia a través de la cual se desarrollan los procesos de combinación, socialización, exteriorización e interiorización del conocimiento. Los participantes exponen sus ideas y opiniones en foros aportando ejemplos y experiencias, así como referencias bibliográficas o documentos que apoyan los argumentos presentados. Para cada foro, un gestor/moderador realiza una síntesis que refleja lo tratado en el debate, elaborando un documento final de propiedad colectiva que es el que se difunde más allá de la propia comunidad.

\subsection{Los grupos de CGC de Tarapacá}

Los grupos de CGC de Tarapacá se conciben como comunidades de profesores vinculados a la UTA que, durante un período de tiempo establecido previamente por la dirección del programa, comparten información, ideas, reflexiones, experiencias y prácticas en torno a un tema de interés común -que es la razón fundamental y constitutiva del mismo grupo. Son:

- Comunidades cerradas, puesto que solo son invitados a participar aquellos profesores que participan en el Programa de Perfeccionamiento en Docencia Universitaria;

- Comunidades virtuales, puesto que la interacción entre los participantes se produce mediante un software de gestión de aprendizaje electrónico;

- Comunidades de carácter temporal, puesto que se crean para alcanzar una meta concreta en un tiempo definido previamente; y

- Comunidades horizontales, puesto que el profesorado participa sin importar su cargo o posición en la institución.

La interacción entre los miembros de la red tiene lugar en el espacio virtual Accelera (http://accelera.uab.cat). Accelera es una plataforma basada en el desarrollo y adecuación de un Course Management System (CMS) de código abierto como Moodle. Se fundamenta en los principios pedagógicos de la construcción social del conocimiento, permitiendo crear y desarrollar comunidades de aprendizaje online, tanto abiertas como cerradas.

Aunque Accelera incluye diversas herramientas de comunicación, la interacción entre el profesorado de la UTA se ha producido básicamente a través del foro. Complementariamente, se han empleado servicios de notificación por correo electrónico, calendario y repositorio documental (ver Figura 4).

Grupos de creación y gestión del conocimiento en red en un programa de perfeccionamiento en docencia universitaria. Anna Díaz-Vicario y Joaquín Gairín Sallán 


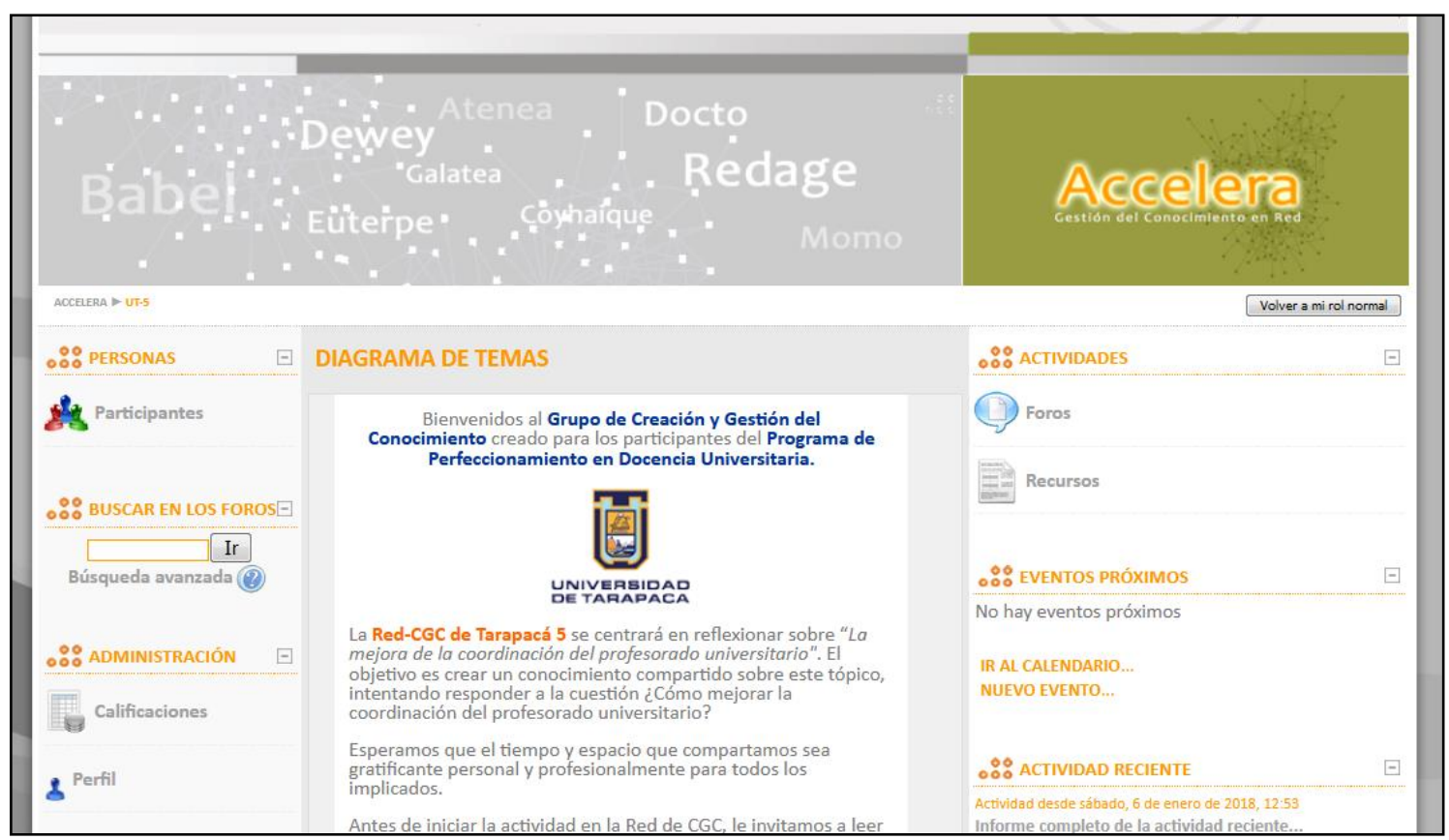

Figura 4. Espacio del Grupo de CGC Tarapacá IV en Accelera.

Dado que en el Programa de Perfeccionamiento han participado hasta un total de 250 profesores, para garantizar un desarrollo óptimo de los grupos de CGC el profesorado se ha distribuido en seis grupos de CGC de entre siete y ocho semanas de duración, desarrollados entre enero de 2016 y junio de 2017.

Cada uno de los grupos se ha centrado a un tema distinto: competencias del profesorado, colaboración y coordinación del profesorado o tutoría académica. En la Tabla 3 puede consultarse el listado de temas tratados y la temporalización de los grupos:

Tabla 3. Temática, pregunta inicial y temporalización de las Redes de CGC.

\begin{tabular}{|l|l|l|}
\hline \multicolumn{1}{|c|}{ Redes de CGC } & \multicolumn{1}{|c|}{ Temáticas } & \multicolumn{1}{|c|}{ Temporalización } \\
\hline Tarapacá I & Las competencias evaluativas del & Del 29/01/2016 al \\
& profesorado universitario & $03 / 04 / 2016$ \\
\hline Tarapacá II & La colaboración docente entre el & Del 06/06/2016 al \\
& profesorado universitario & $27 / 07 / 2016$ \\
\hline Tarapacá III & La competencia digital del & Del 29/07/2016 al \\
& profesorado universitario & $15 / 09 / 2016$ \\
\hline Tarapacá IV & La calidad de la docencia: el rol & Del 14/10/2016 al \\
& del profesorado universitario & $05 / 12 / 2016$ \\
\hline Tarapacá V & La coordinación del profesorado & Del 23/01/2017 al \\
& universitario & $15 / 03 / 2017$ \\
\hline Tarapacá VI & La tutoría académica en la & Del 04/05/2017 al \\
& universidad & $23 / 06 / 2017$ \\
\hline
\end{tabular}

El tema tratado en cada uno de los grupos se ha acordado con los participantes durante una de las sesiones presenciales impartidas por profesorado de la UAB. En primer lugar, los participantes identificaron retos y necesidades del profesorado de la UTA, que posteriormente priorizaron según su importancia y urgencia. Se descartaron aquellos

Grupos de creación y gestión del conocimiento en red en un programa de perfeccionamiento en docencia universitaria. Anna Díaz-Vicario y Joaquín Gairín Sallán 
temas que se focalizaban en un área o especialidad, seleccionando los que tenían un carácter transversal, se vinculaban a contenidos del programa y respondían a las necesidades de implementación del modelo educativo y pedagógico de la UTA.

Los temas seleccionados son cuestiones sobre las que el profesorado puede reflexionar individualmente, pero también son retos que, al afectar a la universidad en su globalidad, no tiene sentido tratar desde áreas de especialización delimitadas. Su mejora pasa por el impulso de procesos de intervención amplios que afectan a toda la institución y que implican a todo el profesorado independiente de su área, departamento o facultad.

\section{Dinámica de funcionamiento del grupo de CGC}

La dinámica de funcionamiento de los grupos de CGC, aunque versen sobre diferentes temáticas, es el mismo para todos ellos. El proceso habitual es el que sigue: una vez realizada la sesión presencial, todos los participantes reciben una carta formal de presentación en la que se recogen las orientaciones para acceder y darse de alta en la plataforma Accelera, presentándoles también el esquema de trabajo general. Se destina una semana a que los participantes se den de alta, se familiaricen con el funcionamiento de la plataforma y lean algunos documentos adicionales que les ayudan a introducirse en la dinámica de trabajo. Pasado este tiempo, se inicia la secuencia de trabajo (ver un ejemplo en la Tabla 4).

Tabla 4. Ejemplo de secuencia de trabajo para el Grupo de CGC Tarapacá IV.

\begin{tabular}{|c|c|c|c|c|}
\hline Foro & Temática & $\begin{array}{l}\text { Objeto de } \\
\text { análisis }\end{array}$ & Herramientas & Temporización \\
\hline 1 & $\begin{array}{l}\text { ¿Qué entendemos por } \\
\text { calidad de la docencia } \\
\text { universitaria? }\end{array}$ & $\begin{array}{l}\text { Delimitar el } \\
\text { concepto de } \\
\text { calidad de la } \\
\text { docencia }\end{array}$ & \multirow{5}{*}{$\begin{array}{l}\text { Foro }+ \\
\text { aportaciones }\end{array}$} & $\begin{array}{l}\text { Del } 14 \text { al } 23 \text { de } \\
\text { octubre }\end{array}$ \\
\hline 2 & $\begin{array}{l}\text { ¿Qué caracteriza una } \\
\text { docencia de calidad? }\end{array}$ & $\begin{array}{l}\text { Caracterizar la } \\
\text { calidad de la } \\
\text { docencia }\end{array}$ & & $\begin{array}{l}\text { Del } 24 \text { de } \\
\text { octubre al } 3 \text { de } \\
\text { noviembre }\end{array}$ \\
\hline 3 & $\begin{array}{l}\text { ¿Qué indicadores nos } \\
\text { señalarían que hay } \\
\text { calidad docente? }\end{array}$ & $\begin{array}{l}\text { Delimitar } \\
\text { indicadores de } \\
\text { diagnóstico } \\
\end{array}$ & & $\begin{array}{l}\text { Del } 4 \text { al } 13 \text { de } \\
\text { noviembre }\end{array}$ \\
\hline 4 & $\begin{array}{l}\text { ¿Qué déficits podemos } \\
\text { encontrar en la calidad } \\
\text { de la docencia } \\
\text { universitaria? }\end{array}$ & $\begin{array}{l}\text { Identificar } \\
\text { problemáticas y } \\
\text { carencias }\end{array}$ & & $\begin{array}{l}\text { Del } 14 \text { al } 23 \text { de } \\
\text { noviembre }\end{array}$ \\
\hline 5 & $\begin{array}{l}\text { ¿Cómo podemos } \\
\text { solucionar los déficits } \\
\text { encontrados en la } \\
\text { calidad de la docencia } \\
\text { universitaria? }\end{array}$ & $\begin{array}{l}\text { Delimitar } \\
\text { alternativas y } \\
\text { proponer } \\
\text { soluciones }\end{array}$ & & $\begin{array}{l}\text { Del } 24 \text { de } \\
\text { noviembre al } 5 \\
\text { de diciembre }\end{array}$ \\
\hline
\end{tabular}

La temática seleccionada se aborda a través de una dinámica de trabajo progresiva basada en la respuesta a preguntas específicas. En una primera fase, los participantes delimitan e identifican de manera unívoca el concepto central en torno al que gira la

Grupos de creación y gestión del conocimiento en red en un programa de perfeccionamiento en docencia universitaria. Anna Díaz-Vicario y Joaquín Gairín Sallán 
discusión (Foro 1 y 2). Posteriormente, definen indicadores (Foro 3) que les permiten, en el foro posterior, diagnosticar la realidad e identificar problemas y carencias (Foro 4). El diagnóstico colectivo permite a los participantes debatir y concretar alternativas de solución (Foro 5). De este modo, las aportaciones de foros previos sirven de base y nutren las aportaciones posteriores.

Los foros permanecen abiertos entre 10 y 15 días y todos siguen el mismo esquema de trabajo:

1. Apertura del foro temático con indicación de la temporalización, la pregunta de debate y el objetivo semanal.

2. Creación del espacio para el debate "foro" y mensaje de bienvenida a los participantes.

3. Envío de un correo electrónico a todos los participantes en el que se les informa del inicio de la actividad y se les anima a participar.

4. Tres días antes del cierre del foro, envío de un nuevo correo electrónico a los participantes, con avance de las aportaciones realizadas. Si es necesario, se realizan nuevos recordatorios.

5. Cierre del foro.

6. Elaboración del documento de síntesis. Las aportaciones de los participantes son resumidas por el moderador de la red y presentadas de nuevo al grupo para su aprobación. La síntesis se envía por correo electrónico, junto con el mensaje de apertura de un nuevo foro temático. El documento también está disponible en el espacio Accelera.

7. Re-inicio del proceso con la apertura de un nuevo foro temático.

Las síntesis de cada foro sólo se difunden entre los participantes en la red, mientras que el documento de síntesis final se difunde entre todo el profesorado participante en el Programa, así como entre los responsables institucionales de la UTA, quienes han valorado la calidad de las aportaciones hechas por su profesorado.

\section{Análisis del funcionamiento de los grupos de CGC de la UTA}

Los Grupos de CGC en Red, creados en el marco del Programa de Perfeccionamiento en Docencia Universitaria de la UTA, han comportado la creación de comunidades, si atendemos a la definición de Murua, Cacheiro y Gallego (2014), quienes definen la comunidad "como un grupo de personas que interaccionan socialmente porque comparte, temporal o permanentemente, una serie de intereses comunes" (p.2). En esta experiencia, pues, se han conformado comunidades virtuales de aprendizaje temporales, puesto que han sido conformadas por profesores de la misma institución, se han vinculado a un programa formativo y su existencia se ha prolongado hasta que se ha concluido el plan de trabajo.

Los seis grupos de CGC de la UTA han seguido la secuencia de trabajo establecida en el diseño. Todos los grupos han seguido las fases por las que habitualmente pasa todo grupo de CGC, aunque intercambiando el orden entre la fase previa y la fase de planificación. En nuestro caso, la fase de planificación se ha producido antes de la fase previa, que se define como de acceso, motivación y socialización de los participantes. La fase de desarrollo, en la que ha tenido el lugar el proceso de compartir y generar

Grupos de creación y gestión del conocimiento en red en un programa de perfeccionamiento en docencia universitaria. Anna Díaz-Vicario y Joaquín Gairín Sallán 
conocimiento entre todos los participantes, ha respetado, en la mayoría de los casos, los tiempos establecidos. En algunos grupos y para algunos foros, sobre todo los destinados a la definición de indicadores y detección de disfunciones, el moderador ha tenido que tomar la decisión de alargar 2/3 días el proceso con la finalidad de aumentar el número de aportaciones hechas por los participantes. En todos los grupos, el proceso se ha concluido entre 5 y 10 días después de cerrar el quinto foro y una vez elaborado el documento final de síntesis.

Si atendemos al perfil del profesorado implicado en los grupos de CGC, se distinguen hasta tres agentes con perfiles, roles y funciones determinadas: director, dinamizador y participantes. El director de los grupos de CGC coincide, en nuestro caso, con el director del Programa formativo de la UAB. Ha sido el encargado de motivar y estimular al profesorado a participar, dinamizando el proceso de elección de las temáticas a debatir. Ello ha posibilitado que el profesorado participante asumiera los grupos de CGC como una actividad relevante y vinculada a los módulos formativos que configuran el programa.

El dinamizador de los grupos de CGC ha sido un profesor de la UAB que no ha participado en las actividades presenciales. El dinamizador ha actuado tanto de facilitador TIC como de moderador. Como facilitador TIC, ha realizado las gestiones pertinentes para que la plataforma Accelera estuviera a punto para los participantes y ha apoyado a los participantes en el proceso de inscripción y participación en los grupos desde un punto de vista técnico. Como moderador, ha sido el encargado de abrir y cerrar los foros de debate, pautar los procesos, supervisar el desarrollo del debate, recordar las normas de participación (netiquetas), intervenir para reconducir la discusión -en caso de que los participantes se desviaran del tema- y sintetizar y sistematizar el conocimiento generando en el seno de cada grupo. También ha estimulado la participación, reafirmando positivamente las contribuciones hechas y reprobando el silencio.

Por último, los participantes voluntarios, que no son otros que el profesorado de la UTA inscrito en el Programa de Perfeccionamiento, han sido invitados a participar, como mínimo, en alguno de los grupos, pero no todos han adquirido el compromiso de registrarse y participar en el grupo de CGC (ver Figura 3.). La participación ha sido más baja de la que cabría esperar. Globalmente se ha situado en torno al $40 \%$, llegando al $34 \%$ en el caso de Tarapacá III. El porcentaje de participación más alto, aunque con un número de participantes potenciales menor, se registró en el grupo Tarapacá IV.

Grupos de creación y gestión del conocimiento en red en un programa de perfeccionamiento en docencia universitaria. Anna Díaz-Vicario y Joaquín Gairín Sallán 


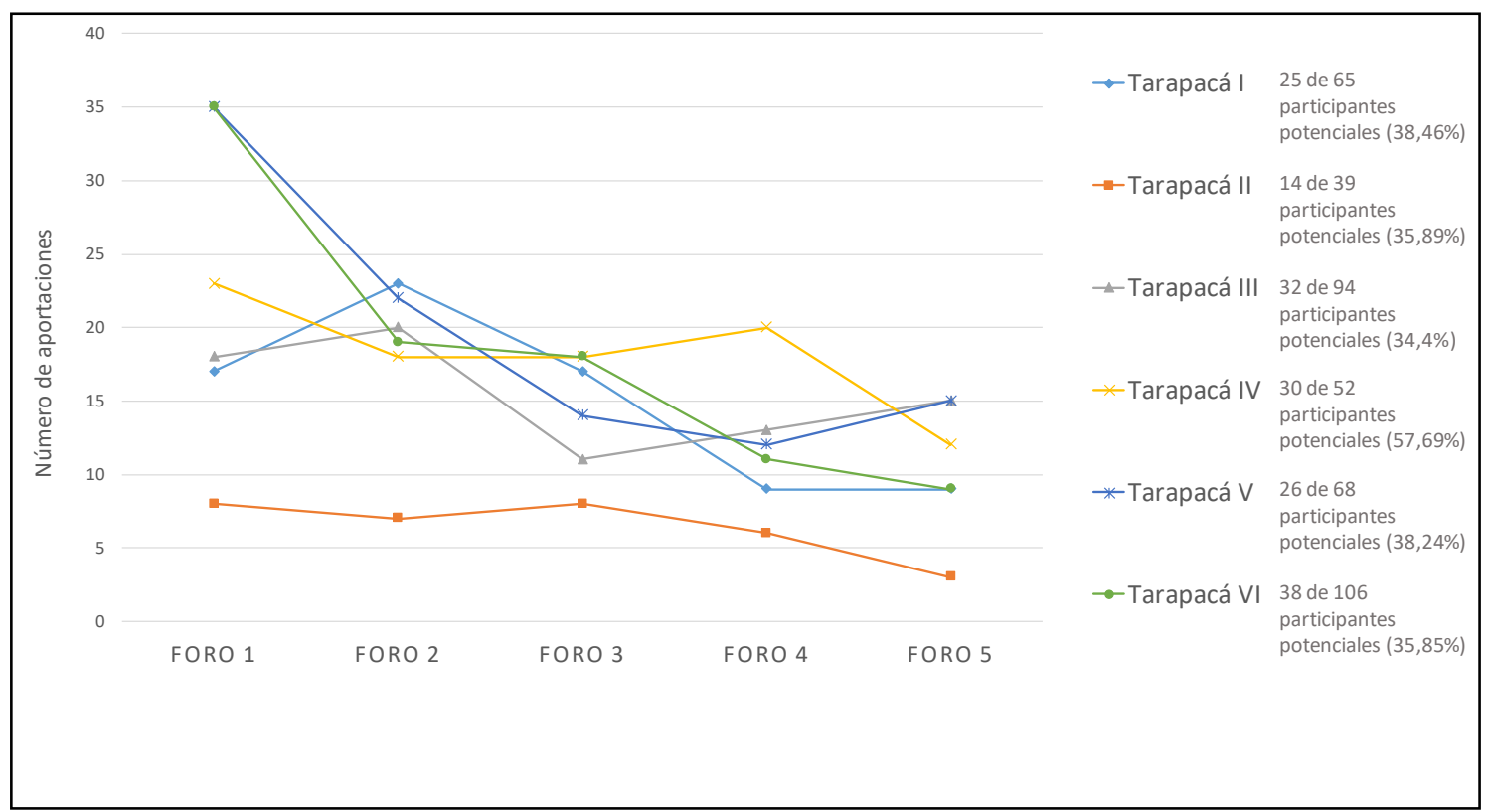

Figura 3. Evolución de la participación en los grupos de CGC de la UTA.

Analizando el nivel de participación por foros, en todos los grupos se han producido dos fenómenos. Por un lado, las aportaciones hechas por los participantes se concentraron en dos momentos: los dos primeros días de inicio del foro y el día posterior al envío del correo recordatorio. Por otro lado, la participación en los foros ha decaído a medida que avanzaban las semanas, aunque algunos han registrado un leve repunte en el cuarto o el quinto foro. El punto álgido de participación ha coincidido en los dos primeros foros destinados a definir unívocamente el concepto y en los que los participantes pueden recurrir a bibliografía existente sobre el tema -requieren un menor grado de abstracción y elaboración. Cabe considerar, al respecto, que los participantes deben de compaginar su alta dedicación docente, con las exigencias del programa formativo y con la participación en el grupo de CGC.

En cuanto a la implicación del profesorado, cabe destacar que, al igual que sucede en otras experiencias (Rodríguez-Gómez, Gairín \& Armengol, 2011), se han conformado tres subgrupos: un grupo central activo, un grupo periférico con participaciones más esporádicas y lurkers o participantes pasivos. El grupo central activo ha realizado, como mínimo, una aportación por foro acompañada de referencias externas y/o ha recapitulado intervenciones de otros compañeros (ver ejemplo en Figura 5).

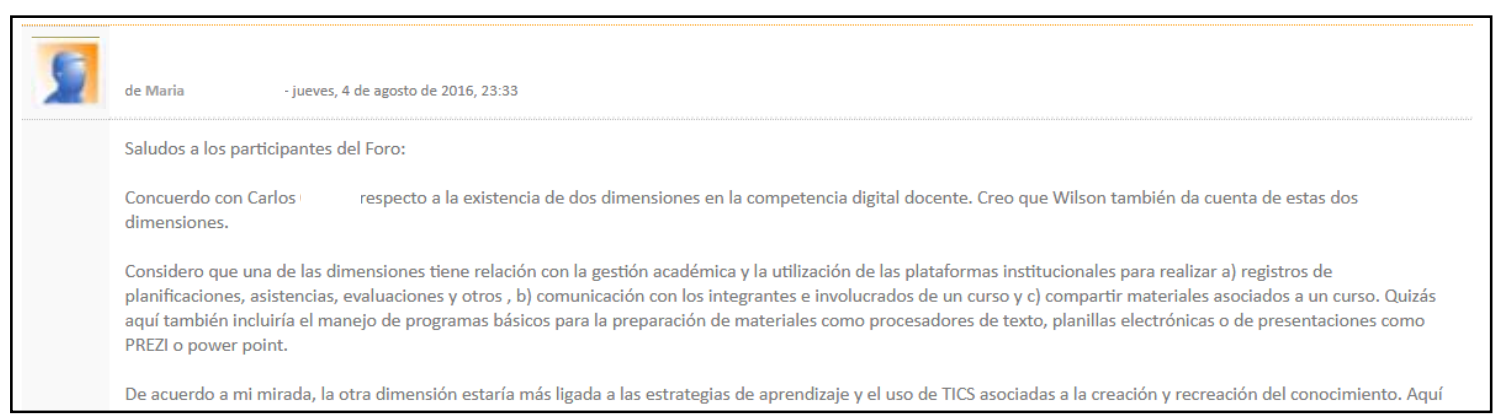

Figura 5. Ejemplo de aportaciones de participante activo (Grupo CGC Tarapacá III, 1r foro).

Grupos de creación y gestión del conocimiento en red en un programa de perfeccionamiento en docencia universitaria. Anna Díaz-Vicario y Joaquín Gairín Sallán 
El grupo periférico ha participado en un máximo de 3 foros, realizando habitualmente reflexiones personales que, ocasionalmente, han apoyado o rebatido puntos de vista de otros colegas. Los participantes pasivos han accedido esporádicamente y no todos han acabado interviniendo en los foros $\mathrm{y}$, en caso de hacerlo, solo han expuesto su punto de vista (ver ejemplo en figura 6). De todas formas, el seguimiento establecido de las actividades del Módulo formativo permite constatar que este colectivo ha seguido los debates y se ha bajado los documentos finales de síntesis.

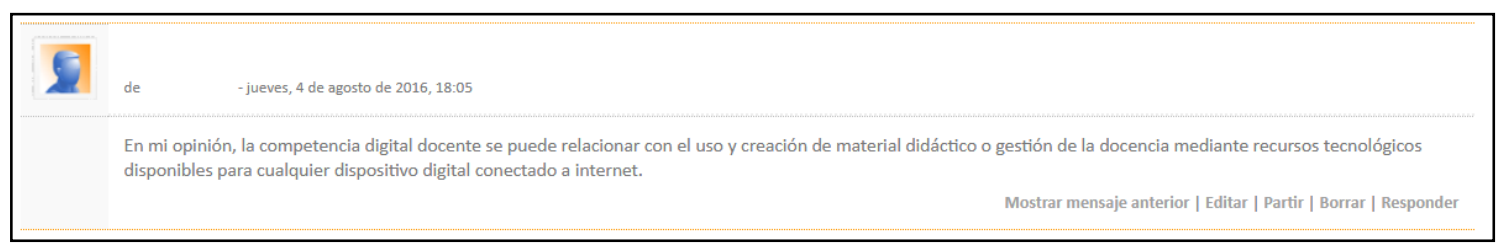

Figura 6. Ejemplo de aportaciones de participante esporádico/pasivo (Grupo CGC Tarapacá III, $1 \mathrm{r}$ foro).

La valoración de la experiencia es positiva, tanto por parte del grupo impulsor, como del profesorado participante y de los responsables institucionales. El profesorado manifestó que la participación en uno o varios grupos de CGC fue una actividad provechosa tanto desde un punto de vista profesional como personal. Destacaron la oportunidad que ofrecen los grupos para generar y compartir conocimiento.

"Muchas gracias por la oportunidad de trabajar en este sistema de foros. Pese a que mi participación no fue como yo quería, creo totalmente que estas oportunidades debieran estar siempre ya que es una gran forma de poder generar y compartir conocimiento." (Participante, Tarapacá I)

Los documentos de síntesis fruto del trabajo en las redes fueron presentados a los responsables institucionales de la UTA, quienes resaltaron el valor de las aportaciones realizadas por su profesorado.

Como puntos clave del desarrollo de la experiencia destacamos:

- La temática central de la red ha surgido de la discusión de los propios participantes.

- La existencia de directrices únicas y claras para gestionar el proceso.

- La presencia de un dinamizador que ha brindado apoyo técnico a los participantes y que ha organizado y coordinado el proceso, hilvanando el debate a través de recapitulaciones y síntesis.

- El establecimiento de una secuencia de trabajo con fecha de inicio y fin, y que ha sido respetada tanto por el dinamizador como por los participantes.

- El respeto a las normas de comportamiento y comunicación de la comunidad virtual.

- La identificación tanto del dinamizador como de los participantes (fotografía, nombre y apellidos y ciudad de origen).

- La generación de un clima de trabajo no competitivo y basado en la comunicación horizontal.

- El uso del correo electrónico para asegurar la correcta coordinación de las actividades y alentar la participación.

Grupos de creación y gestión del conocimiento en red en un programa de perfeccionamiento en docencia universitaria. Anna Díaz-Vicario y Joaquín Gairín Sallán 
Con todo, el análisis del funcionamiento de los grupos de CGC de la UTA revela algunos puntos débiles o áreas de mejora, que necesariamente deben analizarse más en profundidad con el objetivo de solventarlos de cara a futuras experiencias:

- La participación en cinco de los seis grupos no ha alcanzado el 50\% y ha disminuido a lo largo del proceso.

Las causas de la baja inscripción y posterior participación pueden ser múltiples, pero en ocasiones los participantes han manifestado dificultades para participar por coincidir la actividad en los grupos de CGC con períodos de mucho trabajo (cierre del trimestre o curso), con entregas y actividades del programa formativo o con períodos vacacionales. Cabría ver si una modificación en la temporalización, aumentando el tiempo disponible para intervenir en los foros, podría favorecerla.

- El grupo central activo de todos los grupos de CGC ha sido bastante reducido. Dos pueden ser los motivos: falta de cultura colaborativa en la institución y por la motivación más bien extrínseca por participar en este tipo de iniciativas. Seguramente si la actividad hubiera repercutido en la calificación final del programa formativo, se hubiera alcanzado un porcentaje de participación mayor, pero creemos que este no debe ser el motor de motivación del profesorado en este tipo de actividades.

- La mayoría de los profesores ha adoptado un rol de participación esporádicopasivo, realizando intervenciones que se limitaban a reproducir lo dicho por otros autores o en exponer su punto de vista sin debatir lo expresado por otros compañeros. Este hecho ha provocado que más que un debate se haya generado, en ocasiones, un hilo de intervenciones que recogen posturas individuales. Futuros grupos deberán velar por lograr una mayor interacción entre los participantes.

- La única vía de comunicación del dinamizador con los participantes han sido los foros y el correo electrónico. El hecho de ser personal externo a la UTA no ha permitido solucionar algunos problemas técnicos de acceso y registro de algunos participantes. Pero, por otro lado, el hecho de ser un profesor desvinculado del programa formativo y de la UTA ha evitado influir en el profesorado y en las opiniones expresadas -sobre todo en la fase de análisis de disfunciones.

- Los problemas experimentados por algunos profesores para acceder y registrarse en la plataforma Accelera hacen pensar que la formación en competencias digitales puede ser un paso previo que necesariamente debe producirse antes de proceder a conformar grupos de CGC en Red.

\section{Para concluir}

El establecimiento de redes de profesionales que estudian, crean y comparten conocimiento es un hecho crucial para el llamado aprendizaje a lo largo de la vida. Aprender de y con otros es clave en estos tiempos y las TIC, igual que han provocado un cambio en nuestra vida, han de revolucionar los procesos formativos del profesorado (Murua, Cacheiro \& Gallego, 2014).

La experiencia aquí presentada demuestra que los grupos de CGC, como espacios para la construcción social del conocimiento con apoyo de la red, estimulan la reflexión

Grupos de creación y gestión del conocimiento en red en un programa de perfeccionamiento en docencia universitaria. Anna Díaz-Vicario y Joaquín Gairín Sallán

Página $\mathbf{1 7}$ de $\mathbf{2 0}$ 
individual y colectiva del profesorado. Son un espacio para generar, contrastar y ampliar conocimientos sobre un tema particular que puede trascender al propio colectivo de participantes. Asimismo, contribuyen a generar entre el profesorado un mayor sentimiento de pertenencia institucional, al implicarlos en un proceso de búsqueda compartida de soluciones a dilemas vinculados con la docencia y la gestión universitaria.

La puesta en marcha de grupos de CGC en Red no está exenta de problemas, pero creemos que al ser una experiencia que contribuye tanto al desarrollo profesional y personal del profesorado como al aprendizaje y desarrollo organizativo (Duţă \& Rafailă, 2014; Stewart, 2014), es una práctica que merece la pena contemplar como estrategia de formación del profesorado. Involucrar al profesorado en este tipo de actividades también es un mecanismo para potenciar su formación en prácticas de gestión del conocimiento mediante TIC que pueden transferir a sus prácticas docentes (Cantón \& Ferrero, 2014).

Futuras experiencias deberán tomar en cuenta las limitaciones acaecidas en los grupos de CGC de la UTA, incluyendo en su dinámica de funcionamiento prácticas que permitan evaluar el funcionamiento de la red y la satisfacción del profesorado hacia este tipo de iniciativas.

Presentación del artículo: 30 de enero de 2018

Fecha de aprobación: 16 de marzo de 2018

Fecha de publicación: 31 de marzo de 2018

Díaz-Vicario, A., y Gairín, J. (2018). Grupos de Creación y Gestión del Conocimiento en Red en un Programa de Perfeccionamiento en Docencia Universitaria. RED. Revista de Educación a Distancia, 57(5). Consultado el (dd/mm/aaaa) en http://www.um.es/ead/red/57/diaz_gairin.pdf

\section{Financiación}

Esta investigación no ha recibido ninguna subvención específica de los organismos de financiación en los sectores públicos, comerciales o sin fines de lucro.

\section{Referencias bibliográficas}

Ali, O.F., Ghoneim, A., \& Al Roubaie, A. (2014). Knowledge sharing culture in higher education institutions: critical literature review. European, Mediterranean \& Middle Eastern Conference on Information Systems 2014, 1-18.

Avello, R., \& Duart, J.M. (2016). Nuevas tendencias de aprendizaje colaborativo en elearning. Claves para su implementación efectiva. Estudios pedagógicos, 42(1), 271-282.

Grupos de creación y gestión del conocimiento en red en un programa de perfeccionamiento en docencia universitaria. Anna Díaz-Vicario y Joaquín Gairín Sallán 
Bozu, Z., \& Imbernón, F. (2016). El presente y el futuro de la formación permanente docente del profesorado universitario. Educación y ciencia, 5(45), 94-105.

Brunner, J.J., \& Miranda, D.A. (2016). Educación Superior en Iberoamérica. Informe 2016. Santiago de Chile: Centro Interuniversitario de Desarrollo (CINDA). Recuperado de http://www.cinda.cl/wp-content/uploads/2016/11/CINDA-2012Informe-de-Educaci\%C3\%B3n-Superior-INTERIOR-101-1.pdf

Cantón, I., \& Ferrero, E. (2014). La gestión del conocimiento en estudiantes de magisterio. Tendencias pedagógicas, 24, 307-326.

Charband, Y., \& Jafari, N. (2016). Online knowledge sharing mechanisms: a systematic review of the state of the art literature and recommendations for future research. Information Systems Frontiers, 18(6), 1131-1151.

Duţă, N., \& Rafailă, E. (2014). Importance of the lifelong learning for professional development of university teachers - needs and practical implications. ProcediaSocial and Behavioral Sciences, 127, 801-806. DOI: 10.1016/j.sbspro.2014.03.358

Espejo, R.M., \& González, J.M. (2014). El desarrollo de profesores universitarios y escolares en Chile: ¿brecha o continuidad? Perspectiva Educacional. Formación de Profesores, 53(2), 3-19. DOI: 10.4151/07189729-Vol.53-Iss.2-Art.246

Fidalgo-Blanco, Á., Sein-Echaluce, M. L., \& García-Peñalvo, F. J. (2014). Knowledge Spirals in Higher Education Teaching Innovation. International Journal of Knowledge Management, 10(4), 16-37. DOI: 10.4018/ijkm.2014100102

Gairín, J. (2008). La gestión del conocimiento de los directivos. La experiencia virtual de la Red Atenea. Avances en Supervisión Educativa, 8, 1-14.

Gairín, J. (2011). El trabajo colaborativo en red. Actores y procesos en la creación y gestión del conocimiento colectivo. Barcelona: Editorial: DaVinci Continental.

Gairín, J., \& Rodríguez-Gómez, D. (2008). La formación permanente de directivos escolares mediante procesos de creación y gestión del conocimiento. Guía para la gestión de centros educativos (on-line).

Gairín, J., \& Armengol, C. (2006). La Red Atenea para la gestión del conocimiento. Temáticos escuela, 18, 19-22.

Gairín, J., \& Rodríguez-Gómez, D. (2010). Fomento del desarrollo profesional mediante redes de creación y gestión del conocimiento: el caso de la Facultad de Educación de la Universidad Autónoma de Barcelona. En P. Carnicero, P. Silva, \& T. Mentado (Coords.), Nuevos retos de la profesión docente. II Seminario Internacional RELFIDO (pp.48-57). Universidad de Barcelona: Grupo de Investigación FODIP. Formación Docente e Innovación Pedagógica.

Grupos de creación y gestión del conocimiento en red en un programa de perfeccionamiento en docencia universitaria. Anna Díaz-Vicario y Joaquín Gairín Sallán 
García, M., \& Gómez, M. (2015). Prácticas de gestión del conocimiento en los grupos de investigación: un estudio de caso. Revista Interamericana de Bibliotecología, $38(1), 13-25$.

Gairín, J., \& Rodríguez-Gómez, D. (2012). La pràctica professional i la seva vinculació amb la creació i gestió del coneixement col·lectiu. Temps d'educació, 42, 269286.

López, S.P., Peón, J.M.M., \& Ordás, C.J.V. (2009). Information technology as an enabler of knowledge management: an empirical analysis. En W.R. King, (Ed.), Knowledge Management and Organizational Learning, Annals of Information System (Vol. 4, pp. 111-129). Springer: Boston, MA.

Macià, M., \& García, I. (2016). Informal online communities and networks as a source of teacher professional development. A review. Teaching and Teacher Education, 55, 291-307. DOI: 10.1016/j.tate.2016.01.021

Martínez, A.B., \& Cambiano, R. (2003). Colaborando en la red. Ponencia presentada en Edutec 03. Recuperado de http://gte.uib.es/cd_edutec_2003/pareastematicas.htm

Ministerio de Educación, Cultura y Deporte de España [MEC] (2015). El profesorado del siglo XXI. XXI Encuentro de Consejos Escolares Autonómicos y del Estado. Madrid: Secretaría general Técnica. Subdirección General de Documentación y Publicaciones.

Murua, I., Cacheiro, M. L., \& Gallego, D. J. (2014). Las cibercomunidades de aprendizaje (cCA) en la formación del profesorado. RED, Revista de Educación a Distancia, 43, 1-29.

Puntman, R.T., \& Borko, H. (2000). What do new views of knowledge and thinking have to say about research on teacher learning? Educational Research, 29(1), 415.

Rodríguez-Gómez, D., Gairín, J., \& Armengol, C. (2011). La moderación en el modelo Accelera de creación y gestión del conocimiento. En J. Gairín (coord.), El trabajo colaborativo en red. Actores y proceso en la creación y gestión del conocimiento (pp. 143-160). Barcelona: davinci.

Stewart, C. (2014). Transforming professional development to professional learning. Journal of Adult Education, 43(1), 28-33.

Trust, T., Krutka, D. G., \& Carpenter, J.P. (2016). "Together We Are Better": Professional Learning Networks for Teachers. Computers \& Education, 326, 1534. DOI: 10.1016/j.compedu.2016.06.007

Wenger, E. (1998). Communities of Practice: Learning, Meaning, and Identity. Cambridge, UK: Cambridge University Press.

Grupos de creación y gestión del conocimiento en red en un programa de perfeccionamiento en docencia universitaria. Anna Díaz-Vicario y Joaquín Gairín Sallán 